\title{
Results on Boundary Control for Parabolic Systems Using Backstepping Method
}

\author{
K. Mathiyalagan (D, T. Renugadevi, and A. Shree Nidhi \\ Department of Mathematics, Bharathiar University, Coimbatore 641046, India \\ Correspondence should be addressed to K. Mathiyalagan; kmathimath@gmail.com
}

Received 20 May 2021; Revised 3 November 2021; Accepted 27 December 2021; Published 18 January 2022

Academic Editor: Qingling Wang

Copyright ( $) 2022 \mathrm{~K}$. Mathiyalagan et al. This is an open access article distributed under the Creative Commons Attribution License, which permits unrestricted use, distribution, and reproduction in any medium, provided the original work is properly cited.

\begin{abstract}
This paper focuses on the stabilization problem for the linear parabolic system using the backstepping method. The exponentially stability results for considered parabolic system are derived in two cases with Dirichlet and Neumann local terms. Also, the boundary conditions for the problem is assumed to be mixed or Robin-type boundary conditions. The main aim is to achieve the stability of the considered system using the backstepping method with help of Volterra integral transformation. The explicit solutions of kernel functions in integral transformation is obtained by using Laplace transform and designed a boundary control law to the closed-loop system. Finally, the effectiveness and applicability of the derived results are validated through a singlespecies pattern generation model.
\end{abstract}

\section{Introduction}

The partial differential equations (PDEs) describe the several mathematical models of real-life physical problems such as propagation of heat or sound, chemical reactors, fluid flows, signal processing, population genetics, and many others. The important idea of this work is to study the exponential stability of parabolic systems with mixed or Robin boundary conditions using the backstepping method and boundary control law. This approach provides significant design freedom, a better insight to the key features such as computable transient performance, and ability to handle uncertainties to a certain level. However, it is noted that the well-posedness of explicit solution to kernel equation and appropriate design of the stable target system are the main points to be focused in the process. The considered model has wide range of applications including travelling wavetrains model with oscillatory kinetics, linearizing a tubular chemical adiabatic reactor, single-species pattern generation, and so on. The main aim of this work is to derive some novel results on stabilization of parabolic systems with Dirichlet and Neumann local terms. The stabilization of various types of systems got much attention of the researchers in the existing literature; see [1-3] and references therein.

The boundary control for parabolic PDEs [4-6], for hyperbolic PDEs $[7,8]$ and especially for nonlinear PDEs [9-11] is widely studied using the backstepping method. This method is introduced in $[12,13]$ by constructing an integral operator which maps the solution of heat equation onto solution of linear parabolic equation with analytical coefficients. Earlier, Triggiani started the results for boundary feedback stabilization of PDE [14], and Seidman [15] addressed the results on exact boundary control of parabolic equations. Later, the backstepping technique has been developed by Kokotovic [16] and Lozano and Brogliato [17] for designing stabilizing controls for a special class of nonlinear dynamical systems. The backstepping method is an efficient one to eliminate the destabilizing term while the control is acting only from the boundary [18]. The works in [19-21] addressed the well-posedness of the formulation of parabolic problem under dynamic boundary conditions, stabilization of an unstable heat equation, reaction diffusion-ODE system on convex domains, and heat equation with a heat source at intermediate point, respectively, and boundary control of linear PDEs is extended to plants with nonconstant 
diffusivity/thermal conductivity which has been studied in [22]. The backstepping analysis using the nonnegative semigroup theory for reaction diffusion equation with state delay is investigated in [23].

The backstepping method has been used in real-life models such as the late-lumping control for three different types of PDE such as heat equation, wave equation, and two linear coupled hyperbolic PDEs [24]. A nonlinear feedback controller using backstepping design is addressed in [25]; the results are derived to prove the global asymptotic stabilization of the 1D nonlinear PDE model of unstable burning in a solid-propellant rocket. The results on radially varying reaction coefficients under revolution symmetry conditions on a sphere for unstable linear reaction diffusion equation using boundary control has been solved in [26]. Recently, Ghaderi and Keyanpour [27] studied the backstepping boundary control approach for multidimensional coupled parabolic PDEs.

One-dimensional diffusion-reaction PDE with exotic boundary condition is established in [28] using folding transformation. The combination of the backstepping method and circle criterion to study the stability process for the pool boiling system is established in [29]. Fixed-time stabilization for reaction diffusion system and stabilization of reaction diffusion PDE with delayed distribution actuation are studied in $[30,31]$. Recently, the finite-time bounded control problem for coupled parabolic PDE-ODE systems with time-varying and time-invariant boundary disturbances were obtained in [32]. Besides, a new general decay rate to the wave equation with memory-type boundary oscillations has been established in [33]. Recently, Ghattassi and Laleg [34] analysed the heat transfer in a membrane distillation-based desalination modeled by an advection reaction diffusion coupled at the boundary. The state feedback and output feedback for fixed-time stabilization of a linear parabolic distributed parameter system with spacedependent reactivity is studied in [35]. A generalization of the scalar gradient extremum seeking algorithm, which maximizes static maps in the presence of parabolic PDEs, is presented in [36]. The stabilizability of linear partial integrodifferential equations with local term at left end was studied in [37-39]. From the above motivation, the boundary stabilization for the parabolic system with a local term at the right end is presented in this paper.

First, an exponentially stable target system for the considered unstable parabolic system is derived with help of Volterra integral transformation. The role of integral transformation is to build a change of variables which absorbs the destabilizing terms acting in the domain and allows the boundary control to remove their effect completely with help of backstepping approach. Furthermore, the explicit solution of kernel function of hyperbolic type in the integral transformation is obtained using Laplace transform. Finally, the invertibility of integral transformation is proved to conclude the stability of the closed-loop system through the stability of target system. The main contribution of the paper is listed as follows: (i) The state feedback boundary control design for system with Dirichlet and Neumann local terms (right boundary) is studied

(ii) Main advantage is that the results for mixed and Robin-type boundary conditions can be easily derived from the proposed conditions

(iii) As an application to practical problems, the proposed control design is implemented to a singlespecies pattern generation model.

The paper is organized as follows. Notations and some preliminaries are given at the end of Section 1. The considered linear parabolic system with Dirichlet and Neumann local terms is described in Sections 2 and 3, respectively, which also presents the uniqueness of solution, exponential stability results, and the invertibility of the Volterra integral transformation. A practical application is discussed in Section 4 to show the effectiveness of the proposed results. Finally, some concluding remarks are given in Section 5.

1.1. Notation and Preliminaries. $L^{p}(\Omega)$ is space of measurable functions for which the $p$ th power of the absolute value is Lebesque integrable, $1<p<\infty$; for example, $L^{2}(\Omega)$ is the Hilbert space of square integrable function $u(x, t)$, $x \in(a, b)$ and $t>0 . W^{k, p}(\Omega)$ is Sobolev space which is defined as $\left\{u \in L^{p}(\Omega): D^{\alpha} u \in L^{p}(\Omega), \forall|\alpha| \leq k\right\}$, for example, $W^{1,2}(\Omega)=H^{1}(\Omega) . \mathscr{C}(\Omega)$ is set of all real valued continuous functions defined on the space $\Omega . \mathscr{C}^{2}(a, b)$ is the set of all twice continuously differentiable function in the domain $(a, b) . u_{t}(x, t)$ and $u_{x}(x, t)$ denote the first-order partial derivatives of $u(x, t)$ with respect to $t$ and $x$, and $u_{x x}(x, t)$ denotes the second-order partial derivative of $u(x, t)$ with respect to $x$.

\section{Stabilization of the Parabolic System with Dirichlet Local Term}

The diffusion equation is a parabolic PDE; in physics, it describes the macroscopic behavior of many micro-particles in Brownian motion (resulting from the random movements and collisions of the particles). These systems have found many applications ranging from chemical and biological phenomena to medicine, genetics, physics, finance, weather prediction, and so on. Consider the linear parabolic system as follows:

$$
\begin{aligned}
w_{t}(x, t)-\varepsilon w_{x x}(x, t) & =c_{1} w(1, t), \\
(1-\alpha) w(0, t)-\alpha w_{x}(0, t) & =U(t), \\
(1-\beta) w(1, t)+\beta w_{x}(1, t) & =0
\end{aligned}
$$

where $x \in(0,1)$ is a spacial variable, $t>0$ is the time, the state variable $w(x, t) \in \mathscr{R}$ describes density/concentration of a substance, second term on the left-hand side describes the "diffusion" with diffusion coefficient $\varepsilon>0, c_{1}$ is an arbitrary constant which comes in the coefficient of Dirichlet local term, $\alpha \in[0,1]$ and $\beta \in(0,1]$ are the constant 
coefficient for boundary conditions and $U(t)$ is the actuation control input placed in the left boundary. The initial condition for considered system is $w(x, 0)=w_{0}(x)$. Figure 1 represents the process of the considered system (1).

Remark 1. It is noted that the values $\alpha, \beta=0, \alpha, \beta=1$, and $\alpha, \beta \in(0,1)$ represent Dirichlet, Neumann, and Robin boundary conditions, respectively. Also, $\beta=0$ has not been taken into account as it affects the local term, that is, $w(1, t)=0$, which vanishes the Dirichlet local term in (1).

The parabolic system (1) with the sources of instabilities term $c_{1}$ when $U(t)=0$ is unstable. Then, we have to recover the instability term using the backstepping method and by designing appropriate control law. For this, we consider the following Volterra integral transformation in an upper triangular domain which is given by

$$
u(x, t)=w(x, t)-\int_{x}^{1} p(x, y) w(y, t) \mathrm{d} y,
$$

where $p(x, y) \in \mathscr{C}^{2}(0,1)$ is kernel function to be found. Now, applying transformation (2) in (1), we get an exponentially stable target system as

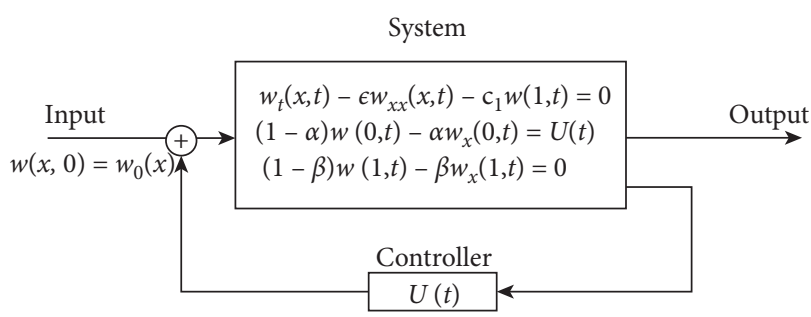

FIGURE 1: Block diagram of system (1).

$$
\begin{aligned}
u_{t}(x, t)-\varepsilon u_{x x}(x, t) & =0 \\
(1-\alpha) u(0, t)-\alpha u_{x}(0, t) & =0 \\
(1-\beta) u(1, t)+\beta u_{x}(1, t) & =0
\end{aligned}
$$

where $\varepsilon>0, \alpha \in[0,1], \beta \in(0,1]$, and $u(x, t) \in \mathscr{R}$ with initial condition $u(x, 0)=u_{0}(x)$.

Next, we take the compatible condition of initial condition in (1) as follows:

$$
\begin{aligned}
& (1-\alpha) w_{0}(0)-\alpha w_{x}(0,0)=\alpha p(0,0) w_{0}(0)+(1-\alpha) \int_{0}^{1} p(0, y) w_{0}(y) \mathrm{d} y-\alpha \int_{0}^{1} p_{x}(0, y) w_{0}(y) \mathrm{d} y \\
& (1-\beta) w_{0}(1)+\beta w_{x}(1,0)=0
\end{aligned}
$$

Definition 1. The system of PDE $u_{t}-\Delta u=f(u)$, where $u: \Omega \longrightarrow \mathscr{R}$ and $\Omega \in(a, b) \times(0, \infty)$ with an initial value $u(x, 0)=u_{0}(x)$ is said to be exponentially stable at the equilibrium $u=0$ if there exist constants $M>0$ and $\alpha>0$ such that

$$
\|u(x, t)\| \leq \mathrm{Me}^{-\alpha t}\|u(x, 0)\|, \quad \forall t \geq 0,
$$

where $\|\cdot\|$ denotes any convenient vector norm.

Lemma 1 (see [40]). Let $u \in W^{1,2}([a, b], \mathscr{R})$ be a scalar function.

Poincare inequality:

$\int_{a}^{b}|u(x, t)|^{2} \mathrm{~d} x \leq(b-a) u^{2}(a, t)+(b-a)^{2} \int_{a}^{b}\left|u_{x}(x, t)\right|^{2} \mathrm{~d} x$.

Poincare-Friedrichs inequality:

$$
\begin{aligned}
\int_{a}^{b}|u(x, t)|^{2} \mathrm{~d} x \leq & (b-a) u^{2}(b, t)+(b-a) u^{2}(a, t) \\
& +(b-a)^{2} \int_{a}^{b}\left|u_{x}(x, t)\right|^{2} \mathrm{~d} x .
\end{aligned}
$$

Now, our main aim here is to prove the exponential stability of considered system (1) and design the boundary control input which will be achieved through main results given below.

2.1. Uniqueness of the Solution of (1). In this section, first, we derive the boundary control input for system (1) using backstepping transformation and then prove the exponential stability of system (3).

Theorem 1. For any $c_{1}$, there exist a function $p(\cdot, \cdot) \in \mathscr{C}^{2}(0,1):\{0 \leq x \leq y \leq 1\}$ such that $w_{0}(\cdot) \in L^{2}(0,1)$; with compatibility conditions (4) and (5), the closed-loop system (1) with feedback controller,

$$
\begin{aligned}
U(t)= & \alpha p(0,0) w(0, t)+(1-\alpha) \int_{0}^{1} p(0, y) w(y, t) \mathrm{d} y \\
& -\alpha \int_{0}^{1} p_{x}(0, y) w(y, t) \mathrm{d} y, \quad \alpha \in[0,1] .
\end{aligned}
$$

has a unique solution $w(x, t)$ in $\mathscr{C}\left([0, \infty) ; L^{2}(0,1)\right)$.

Proof. First, we will show that system (1) is transferable to exponentially stable system (3) by deriving the explicit solution for kernel function. Here, we derived the kernel equation and its explicit solution. First, we differentiate transformation (2) with respect to $x$ and $t$ : 


$$
\begin{aligned}
u_{t}(x, t)= & w_{t}(x, t)+\varepsilon\left(\frac{1-\beta}{\beta}\right) p(x, 1) w(1, t)+p(x, x) \varepsilon w_{x}(x, t)+p_{y}(x, 1) \varepsilon w(1, t) \\
& -p_{y}(x, x) \varepsilon w(x, t)-\int_{x}^{1} p_{y y}(x, y) \varepsilon w(y, t) \mathrm{d} y-\int_{x}^{1} p(x, y) c_{1} w(1, t) \mathrm{d} y \\
u_{x}(x, t)= & w_{x}(x, t)+p(x, x) w(x, t)-\int_{x}^{1} p_{x}(x, y) w(y, t) \mathrm{d} y \\
u_{x x}(x, t)= & w_{x x}(x, t)+\frac{\mathrm{d}}{\mathrm{d} x} p(x, x) w(x, t)+p(x, x) w_{x}(x, t)+p_{x}(x, x) w(x, t)-\int_{x}^{1} p_{x x}(x, y) w(y, t) \mathrm{d} y
\end{aligned}
$$

where $\quad \mathrm{d} / \mathrm{d} x p(x, x)=p_{x}(x, x)+p_{y}(x, x), p_{x}(x, x)=\partial /$ $\left.\partial x p(x, y)\right|_{y=x}, p_{y}(x, x)=\partial /\left.\partial y p(x, y)\right|_{y=x}$

From (10)-(12), we obtain

$$
\begin{aligned}
u_{t}(x, t)-\varepsilon u_{x x}(x, t)= & {\left[-\varepsilon p_{y}(x, x)-\varepsilon \frac{\mathrm{d}}{\mathrm{d} x} p(x, x)-\varepsilon p_{x}(x, x)\right] w(x, t) } \\
& +\left[c_{1}+\varepsilon\left(\frac{1-\beta}{\beta}\right) p(x, 1)+\varepsilon p_{y}(x, 1)-\int_{x}^{1} p(x, y) c_{1} \mathrm{~d} y\right] w(1, t) \\
& +\int_{x}^{1} \varepsilon\left[p_{x x}(x, y)-p_{y y}(x, y)\right] w(y, t) \mathrm{d} y
\end{aligned}
$$

In order to get the target system (3), we choose that the kernel $p(x, y)$ satisfies the following hyperbolic PDE with integral boundary condition. In (13), the first term of righthand side becomes $2 \varepsilon \mathrm{d} / \mathrm{d} x p(x, x)=0$. Then, we obtain

$$
\begin{aligned}
p_{x x}(x, y)-p_{y y}(x, y) & =0, \\
p(x, x) & =0, \\
p_{y}(x, 1)= & -\frac{c_{1}}{\varepsilon}-\left(\frac{1-\beta}{\beta}\right) p(x, 1) \\
& +\frac{c_{1}}{\varepsilon} \int_{x}^{1} p(x, y) \mathrm{d} y,
\end{aligned}
$$

where $0 \leq x \leq y \leq 1$. Next is to find an explicit form of kernel solution $p(x, y)$. Let $\xi=1-x, \quad \eta=1-y$, and $G(\xi, \eta)=p(x, y)$. Now, applying change of variables, the kernel PDE is converted to

$G_{\xi \xi}(\xi, \eta)-G_{\eta \eta}(\xi, \eta)=0$

$$
\begin{aligned}
G(\xi, \xi) & =0 \\
G_{\eta}(\xi, 0) & =\frac{c_{1}}{\varepsilon}+\left(\frac{1-\beta}{\beta}\right) G(\xi, 0)-\frac{c_{1}}{\varepsilon} \int_{0}^{\xi} G(\xi, \tau) \mathrm{d} \tau
\end{aligned}
$$

where $G_{\xi}=-p_{x}, G_{\eta}=-p_{y}, G_{\xi \xi}=p_{x x}$, and $G_{\eta \eta}=p_{y y}$.

It is obvious that the homogeneous equation in (15) has a general solution of the form $G(\xi, \eta)=\phi(\xi-\eta)+\psi(\xi+\eta)$, using the boundary condition $G(\xi, \xi)=0$; without loss of generality, we set $\psi \equiv 0$ and $\phi(0)=0$. Therefore,

$$
G(\xi, \eta)=\phi(\xi-\eta)
$$

Next, from integral boundary condition in (15), we have

$$
\phi^{\prime}(\xi)=-\frac{c_{1}}{\varepsilon}-\left(\frac{1-\beta}{\beta}\right) \phi(\xi)-\frac{c_{1}}{\varepsilon} \int_{0}^{\xi} \phi(\mu) \mathrm{d} \mu .
$$
obtain

Applying the Laplace transform with respect to $\xi$, we

$$
\begin{aligned}
s \Phi(s)-\phi(0) & =-\frac{c_{1}}{\varepsilon} \frac{1}{s}-\left(\frac{1-\beta}{\beta}\right) \Phi(s)+\frac{c_{1}}{\varepsilon} \frac{\Phi(s)}{s}, \\
\Phi(s) & =-\frac{c_{1}}{\varepsilon}\left(\frac{1}{s^{2}+(1-\beta / \beta) s-c_{1} / \varepsilon}\right) \\
& =-\frac{c_{1}}{\varepsilon}\left(\frac{1}{s_{1}-s_{2}}\right)\left[\frac{1}{s-s_{1}}-\frac{1}{s-s_{2}}\right],
\end{aligned}
$$

where $s_{1}=-(1-\beta / 2 \beta)+\sqrt{(1-\beta / 2 \beta)^{2}+c_{1} / \epsilon}$ and $s_{2}=$ $-(1-\beta / 2 \beta)-\sqrt{(1-\beta / 2 \beta)^{2}+c_{1} / \epsilon}$.

Now, applying inverse Laplace transform gives

$$
\phi(z)=-\frac{c_{1}}{\varepsilon}\left(\frac{1}{s_{1}-s_{2}}\right)\left[e^{s_{1} z}-e^{s_{2} z}\right]
$$

Thus, we have

$$
G(\xi, \eta)=\phi(\xi-\eta)=-\frac{c_{1}}{\varepsilon}\left(\frac{1}{s_{1}-s_{2}}\right)\left[e^{s_{1}(\xi-\eta)}-e^{s_{2}(\xi-\eta)}\right] .
$$


Hence, from $G(\xi, \eta)=p(x, y)$, the explicit solution of kernel PDE (14) is given by

$$
p(x, y)=-\frac{c_{1}}{\varepsilon}\left(\frac{1}{s_{1}-s_{2}}\right)\left[e^{s_{1}(y-x)}-e^{s_{2}(y-x)}\right] .
$$

which is continuously differentiable in $[0,1]$.

Remark 2. The structure of the left boundary controller is in the form as follows, using integral transformation (2):

(i) For $\alpha=0$, then the left boundary controller becomes Dirichlet boundary feedback controller, that is, $w(0, t)=U(t)$ and $u(0, t)=0$ :

$$
U(t)=\int_{0}^{1} p(0, y) w(y, t) \mathrm{d} y .
$$

(ii) For $\alpha=1$, then the left boundary controller becomes the Neumann boundary feedback controller, that is, $w_{x}(0, t)=-U(t)$ and $u_{x}(0, t)=0$ :

$$
U(t)=p(0,0) w(0, t)-\int_{0}^{1} p_{x}(0, y) w(y, t) \mathrm{d} y .
$$

(iii) For $\alpha \in(0,1)$, then the left boundary controller becomes the Robin boundary feedback controller, that is, $\quad(1-\alpha) w(0, t)-\alpha w_{x}(0, t)=U(t) \quad$ and $(1-\alpha) u(0, t)-\alpha u_{x}(0, t)=0$ :

$$
\begin{aligned}
U(t)= & \alpha p(0,0) w(0, t)+(1-\alpha) \int_{0}^{1} p(0, y) w(y, t) \mathrm{d} y \\
& -\alpha \int_{0}^{1} p_{x}(0, y) w(y, t) \mathrm{d} y .
\end{aligned}
$$
by

It then follows the boundary control input which is given

$$
\begin{aligned}
w(0, t)= & U(t)=\frac{c_{1}}{\varepsilon}\left(\frac{1}{s_{1}-s_{2}}\right) \int_{0}^{1}\left[e^{s_{1} y}-e^{s_{2} y}\right] w(y, t) \mathrm{d} y, \\
w_{x}(0, t)= & -U(t)=-\frac{c_{1}}{\varepsilon}\left(\frac{1}{s_{1}-s_{2}}\right) \int_{0}^{1}\left[-s_{1} e^{s_{1} y}+s_{2} e^{s_{2} y}\right] w(y, t) \mathrm{d} y, \\
(1-\alpha) w(0, t)-\alpha w_{x}(0, t)= & U(t)=-(1-\alpha) \frac{c_{1}}{\varepsilon}\left(\frac{1}{s_{1}-s_{2}}\right) \int_{0}^{1}\left[e^{s_{1} y}-e^{s_{2} y}\right] w(y, t) \mathrm{d} y \\
& +\alpha \frac{c_{1}}{\varepsilon}\left(\frac{1}{s_{1}-s_{2}}\right) \int_{0}^{1}\left[-s_{1} e^{s_{1} y}+s_{2} e^{s_{2} y}\right] w(y, t) \mathrm{d} y, \quad \alpha \in(0,1) .
\end{aligned}
$$

Furthermore, the explicit solution of the target system (3) is possible to get in the simple heat equation form (detailed in [39]). That is,

$$
u(x, t)=\sum_{n=1}^{\infty} A_{n} \Phi_{n}(x) e^{-\lambda_{n}^{2} t}
$$

where $\lambda_{n}=(2 n-1) \pi \sqrt{\varepsilon} / 2$ is the eigenvalue, $\Phi_{n}(x)=(1-$ $\alpha / \alpha) \lambda_{n} / \sqrt{\varepsilon} \cos \left(\lambda_{n} / \sqrt{\varepsilon} x\right)+\sin \left(\lambda_{n} / \sqrt{\varepsilon} x\right)$ is the eigenfunction, and $A_{n}=\int_{0}^{1} u_{0} \Phi_{n}(x) \mathrm{d} x / \int_{0}^{1} \Phi_{n}^{2}(x) \mathrm{d} x$ is the effect of initial conditions. With the Volterra integral transformation, employing the solution of the target system easily gives the solution of system (1).

Therefore, the explicit solution of kernel function exists and compatibility condition with initial value holds. Thus, a unique solution $w(x, t)$ of the closed-loop system (1) exists. This completes the proof.
2.2. Exponential Stability Results for (3). Next, the exponential stability of target system (3) is proved using the Lyapunov stability analysis.

Theorem 2. For $\varepsilon>0$ and $\beta \in(0,1]$, the target system (3) with mixed boundary conditions at $\alpha=0$ or $\alpha=1$ for arbitrary initial condition $u_{0}(x) \in L^{2}(0,1)$ is an exponentially stable in $L^{2}(0,1)$ if the following inequality holds

$$
\|u(x, t)\|_{L^{2}} \leq e^{-\varepsilon t}\left\|u_{0}(x)\right\|_{L^{2}} .
$$

Under the condition, $(1-\beta / \beta) \geq 0$.

Proof. To prove the stability of (3), consider the Lyapunov function as follows:

$$
V(t, u(x, t))=\frac{1}{2} \int_{0}^{1} u^{2}(x, t) \mathrm{d} x .
$$


Since $u(x, t)$ is a differentiable function, taking time derivative and using boundary condition in (3) and integration by parts, we obtain

$$
\begin{aligned}
\frac{\partial V(\cdot)}{\partial t} & =\int_{0}^{1} u(x, t) u_{t}(x, t) \mathrm{d} x \\
& =\int_{0}^{1} u(x, t) \varepsilon u_{x x}(x, t) \mathrm{d} x \\
& =\varepsilon u(1, t) u_{x}(1, t)-\varepsilon u(0, t) u_{x}(0, t)-\int_{0}^{1} \varepsilon u_{x}^{2}(x, t) \mathrm{d} x \\
& =-\varepsilon\left(\frac{1-\beta}{\beta}\right) u^{2}(1, t)-\int_{0}^{1} \varepsilon u_{x}^{2}(x, t) \mathrm{d} x
\end{aligned}
$$

Using inequality in Lemma 1, yields that

$$
\frac{\partial V(\cdot)}{\partial t} \leq-\varepsilon \int_{0}^{1} u^{2}(x, t) \mathrm{d} x .
$$

In view of (18), we have $\partial V(\cdot) / \partial t \leq-2 \varepsilon V(t, u(x, t))$. Solving this inequality, we obtain

$$
V(t, u(x, t)) \leq e^{-2 \varepsilon t} V(0, u(x, 0)),
$$

where $V(0, u(x, 0))=1 / 2 \int_{0}^{1} u_{0}^{2}(x) \mathrm{d} x$. Therefore, from (30), we get inequality (29). Hence, from Definition 1, it can be concluded that the target system is an exponential stability in $L^{2}(0,1)$. This completes the proof.

Suppose if $\alpha \in(0,1)$, the boundary condition of target system (3) turned to be Robin type, the stability results for this case will be proved in the following theorem.

Theorem 3. For $\varepsilon>0$ and $\beta \in(0,1]$, the target system (3) under Robin boundary conditions at $\alpha \in(0,1)$ for arbitrary initial condition $u_{0}(x) \in L^{2}(0,1)$ is an exponentially stable in $L^{2}(0,1)$ if the following inequality holds

$$
\|u(x, t)\|_{L^{2}} \leq e^{-\epsilon t}\left\|u_{0}(x)\right\|_{L^{2}},
$$

under the condition $(1-\beta / \beta) \geq 0$ and $(1-\alpha / \alpha)>0$.

Proof. Consider the Lyapunov function as given in Theorem 2 , and using the same procedure, we have

$$
\begin{aligned}
\frac{\partial V(\cdot)}{\partial t}= & -\epsilon\left(\frac{1-\beta}{\beta}\right) u^{2}(1, t)-\epsilon\left(\frac{1-\alpha}{\alpha}\right) u^{2}(0, t) \\
& -\int_{0}^{1} \epsilon u_{x}^{2}(x, t) \mathrm{d} x .
\end{aligned}
$$

Applying inequality in Lemma 1, yields

$$
\frac{\partial V(\cdot)}{\partial t} \leq-\varepsilon \int_{0}^{1} u^{2}(x, t) \mathrm{d} x .
$$

In view of (30), we have $\partial V(\cdot) / \partial t \leq-2 \epsilon V(t, u(x, t))$. Integrating the above inequality results,

$$
V(t, u(x, t)) \leq e^{-2 \epsilon t} V(0, u(x, 0))
$$

where $V(0, u(x, 0))=1 / 2 \int_{0}^{1} u_{0}^{2}(x) \mathrm{d} x$. Therefore, from (30), we get inequality (34). Hence, from Definition 1 , it can be concluded that the target system is an exponential stability in $L^{2}(0,1)$. This completes the proof.

2.3. Inverse Transformation for (2). Now, we are in a position to prove the exponential stability of the closed-loop system (1). For this, it is enough to show that transformation (2) is invertible, which can guarantee that the stability of the target system (3) implies that the stability of the closed-loop system (1). Assume the inverse of transformation (2) in the form

$$
w(x, t)=u(x, t)+\int_{x}^{1} q(x, y) u(y, t) \mathrm{d} y,
$$

where $q(x, y) \in \mathscr{C}^{2}(0,1)$ is a kernel function. By following similar procedure in (10)-(13), we obtain a PDE in kernel function as

$$
\begin{aligned}
q_{x x}(x, y)-q_{y y}(x, y) & =0, \\
q(x, x) & =0, \\
q_{y}(x, 1) & =-\frac{c_{1}}{\varepsilon}-\left(\frac{1-\beta}{\beta}\right) q(x, 1) .
\end{aligned}
$$

PDE (39) is similar to (14); using similar lines, the explicit solution of (39) can be achieved.

Suppose transformation (2) and its inverse (20) are mutual transformation pair (inverse to each other), then $p(x, y)$ and $q(x, y)$ should satisfy

$$
q(x, y)=p(x, y)+\int_{x}^{y} p(x, s) q(s, y) \mathrm{d} s .
$$

To prove the required result, we consider

$$
F(x, y)=q(x, y)-p(x, y)-\int_{x}^{y} p(x, s) q(s, y) \mathrm{d} s .
$$

From (14) and (39), we have $F(x, x)=0$. Differentiating (41) with respect to $x$ and $y$, we obtain 


$$
\begin{aligned}
F_{x}(x, y)= & q_{x}(x, y)-p_{x}(x, y)+p(x, x) q(x, y)-\int_{x}^{y} p_{x}(x, s) q(s, y) \mathrm{d} s, \\
F_{y}(x, y)= & q_{y}(x, y)-p_{y}(x, y)-p(x, y) q(y, y)-\int_{x}^{y} p(x, s) q_{y}(s, y) \mathrm{d} s, \\
F_{x x}(x, y)= & q_{x x}(x, y)-p_{x x}(x, y)+\frac{\mathrm{d}}{\mathrm{d} x} p(x, x) q(x, y)+p(x, x) q_{x}(x, y)+p_{x}(x, x) q(x, y) \\
& -p_{y}(x, y) q(y, y)+p_{y}(x, x) q(x, y)-p(x, y) q_{x}(y, y)+p(x, x) q_{x}(x, y)-\int_{x}^{y} p(x, s) q_{s s}(s, y) \mathrm{d} s, \\
F_{y y}(x, y)= & q_{y y}(x, y)-p_{y y}(x, y)-\frac{d}{\mathrm{~d} y} q(y, y) p(x, y)-p_{y}(x, y) q(y, y)-p(x, y) q_{y}(y, y)-\int_{x}^{y} p(x, s) q_{y y}(s, y) \mathrm{d} s .
\end{aligned}
$$

Then, we obtain

$$
\begin{aligned}
F_{x x}(x, y)-F_{y y}(x, y) & =0, \\
F(x, x) & =0, \\
F_{y}(x, 1) & =0 .
\end{aligned}
$$

Obviously, $F(x, y)=0$ is the unique solution of system (43); therefore, transformation (2) and its inverse (38) are mutual transformation pair. Hence, the transformation (2) is invertible.

There, from the above analysis, it is easy to conclude that the parabolic system with Dirichlet local term (1) is exponentially stable.

\section{Stabilization of the Parabolic System with Neumann Local Term}

Consider the linear system as follows:

$$
\begin{aligned}
w_{t}(x, t)-\varepsilon w_{x x}(x, t) & =c_{2} w_{x}(1, t), \\
(1-\alpha) w(0, t)-\alpha w_{x}(0, t) & =\bar{U}(t), \\
(1-\beta) w(1, t)+\beta w_{x}(1, t) & =0,
\end{aligned}
$$

where $x \in(0,1), t>0, \varepsilon>0, \alpha \in[0,1]$, and $\beta \in(0,1)$, the state variable $w(x, t) \in \mathscr{R}$ with initial condition $w(x, 0)=w_{0}(x), c_{2}$ is an arbitrary constant which describes the coefficient of local term (that is, Neumann interconnection), and $\bar{U}(t)$ is the actuation control input.

Remark 3. Substitute the right boundary condition $w_{x}(1, t)=-(1-\beta / \beta) w(1, t)$ in $(44)$ at the place of Neumann interconnection which is equivalent to system (1). However, it is noted that we will get a different form of the kernel function and boundary inputs for the above system, which is the main reason to proceed with the following results.

For $\bar{U}(t)=0$, the proposed system (44) is unstable one. Then, the stability of this system is derived by using the backstepping transformation. Here, we use the following Volterra integral transformation with upper triangular structure:

$$
u(x, t)=w(x, t)-\int_{x}^{1} l(x, y) w(y, t) \mathrm{d} y,
$$

where $l(x, y) \in \mathscr{C}^{2}(0,1)$ is the kernel function to be found. Now, applying transformation (45) in (44), we get an exponentially stable target system (3).

Then, we set the compatible condition of initial condition in (44) as follows:

$$
\begin{aligned}
& (1-\alpha) w_{0}(0)-\alpha w_{x}(0,0)=\alpha l(0,0) w_{0}(0)+(1-\alpha) \int_{0}^{1} l(0, y) w_{0}(y) \mathrm{d} y-\alpha \int_{0}^{1} l_{x}(0, y) w_{0}(y) \mathrm{d} y, \\
& (1-\beta) w_{0}(1)+\beta w_{x}(1,0)=0 .
\end{aligned}
$$




\subsection{Uniqueness of the Solution of (44)}

Theorem 4. For any $c_{2}$, there exist a function $l(\cdot, \cdot) \in \mathscr{C}^{2}(0,1):\{0 \leq x \leq y \leq 1\}$ such that $w_{0}(\cdot) \in L^{2}(0,1)$; with compatibility conditions (46) and (47), the closed-loop system (44) with the feedback controller,

$$
\bar{U}(t)=\alpha l(0,0) w(0, t)+(1-\alpha) \int_{0}^{1} l(0, y) w(y, t) \mathrm{d} y-\alpha \int_{0}^{1} l_{x}(0, y) w(y, t) \mathrm{d} y, \quad \alpha \in[0,1]
$$

has a unique solution $w(x, t)$ in $\mathscr{C}\left([0, \infty) ; L^{2}(0,1)\right)$.

Proof. First, we find the kernel function and its solution. Substituting the transformation in (45) and then following the same procedure in Theorem 1. We get the kernel function of hyperbolic type:

$$
\begin{aligned}
l_{x x}(x, y)-l_{y y}(x, y) & =0, \\
l(x, x) & =0, \\
l_{y}(x, 1) & =\frac{c_{2}}{\epsilon}\left(\frac{1-\beta}{\beta}\right)-\left(\frac{1-\beta}{\beta}\right) l(x, 1)+\frac{c_{2}}{\epsilon}\left(\frac{1-\beta}{\beta}\right) \int_{x}^{1} l(x, y) \mathrm{d} y .
\end{aligned}
$$

Solving the above kernel equation using change of variables and Laplace transforms, the explicit solution of kernel is

$$
l(x, y)=\frac{c_{2}}{\varepsilon}\left(\frac{1-\beta}{\beta}\right)\left(\frac{1}{s_{3}-s_{4}}\right)\left[e^{s_{3}(y-x)}-e^{s_{4}(y-x)}\right],
$$

where $s_{3}=-(1-\beta / 2 \beta)+\sqrt{(1-\beta / 2 \beta)^{2}-c_{2} / \mathcal{E}(1-\beta / \beta)}$ and $s_{4}=-(1-\beta / 2 \beta)-\sqrt{(1-\beta / 2 \beta)^{2}-c_{2} / \epsilon(1-\beta / \beta)}$.

The left boundary controller in (44) has the same result as in Remark 2. Substituting the kernel solution in the boundary control law is given by

$$
\begin{aligned}
w(0, t)= & \frac{c_{2}}{\varepsilon}\left(\frac{1-\beta}{\beta}\right)\left(\frac{1}{s_{3}-s_{4}}\right) \int_{0}^{1}\left[e^{s_{3} y}-e^{s_{4} y}\right] w(y, t) \mathrm{d} y, \\
w_{x}(0, t)= & -\frac{c_{2}}{\varepsilon}\left(\frac{1-\beta}{\beta}\right)\left(\frac{1}{s_{3}-s_{4}}\right) \int_{0}^{1}\left[-s_{3} e^{s_{3} y}+s_{4} e^{s_{4} y}\right] w(y, t) \mathrm{d} y, \\
(1-\alpha) w(0, t)-\alpha w_{x}(0, t)= & (1-\alpha) \frac{c_{2}}{\varepsilon}\left(\frac{1-\beta}{\beta}\right)\left(\frac{1}{s_{3}-s_{4}}\right) \int_{0}^{1}\left[e^{s_{3} y}-e^{s_{4} y}\right] w(y, t) \mathrm{d} y \\
& -\alpha \frac{c_{2}}{\varepsilon}\left(\frac{1-\beta}{\beta}\right)\left(\frac{1}{s_{3}-s_{4}}\right) \int_{0}^{1}\left[-s_{3} e^{s_{3} y}+s_{4} e^{s_{4} y}\right] w(y, t) \mathrm{d} y .
\end{aligned}
$$

Next, the exponential stability of target system (3) for this case followed from Theorems 2 and 3 with $(1-\beta / \beta)>0$ and $(1-\alpha / \alpha)>0$.

Remark 4. It should be noted that the arguments on $\alpha$ in Remark 1 will hold in this case too. Furthermore, the value of $\beta=0$ and $\beta=1$ are not considered here because, when $\beta=0$ affects the local term and when $\beta=1$, boundary control becomes undefined.
3.2. Inverse Transformation for (45). Now, we are in a position to prove the exponential stability of the closed-loop system (44). For this, it is enough to show that transformation (45) is invertible, which can guarantee that the stability of the target system (3) implies that the stability of the closed-loop system (44). Assume the inverse of transformation (45) in the form

$$
w(x, t)=u(x, t)+\int_{x}^{1} m(x, y) u(y, t) \mathrm{d} y,
$$


where $m(x, y) \in \mathscr{C}^{2}(0,1)$ is a kernel function. By following similar procedure in (10)-(13), we obtain a PDE in kernel function as

$$
\begin{aligned}
m_{x x}(x, y)-m_{y y}(x, y) & =0, \\
m(x, x) & =0, \\
m_{y}(x, 1)= & \frac{c_{2}}{\varepsilon}\left(\frac{1-\beta}{\beta}\right)+\frac{c_{1}}{\varepsilon} m(1,1) \\
& -\left(\frac{1-\beta}{\beta}\right) m(x, 1) .
\end{aligned}
$$

This hyperbolic PDE (53) is similar to (49); using similar lines, the explicit solution of (53) can be achieved. There, from the above analysis, it is easy to conclude that the parabolic system with Neumann local term (44) is exponentially stable.

\section{Applications}

In this section, the single-species pattern generation which has the spatial heterogeneity with the Budworm spruce model [41] will be discussed.

Consider the one-dimensional reaction diffusion system for single-species pattern generation given by

$$
\vartheta_{t}(x, t)-D \vartheta_{x x}(x, t)=f(\vartheta(x, t)),
$$

where $\vartheta(\cdot)$ denotes the population density of a species, $x \in(0, L)$ is the spatial domain for the species, $t>0$ is the time taken for pattern generation, $f(\vartheta(x, t))$ is the species dynamics (possibly linear or nonlinear), $D$ is the measure of the dispersal efficiency of the species, and $\vartheta(x, 0)=\vartheta_{0}(x)$ is the initial population distribution of the species. Generally, the external environment is not always favorable one; that is, there may be some unfavorable circumstances that can be described by appropriate boundary conditions given by

$$
\begin{aligned}
& \kappa \vartheta(0, t)-\vartheta_{x}(0, t)=0, \\
& \kappa \vartheta(L, t)+\vartheta_{x}(L, t)=0,
\end{aligned}
$$

where $\kappa$ is the parameter for a value of the unfavorable situation. It is noted that when $\kappa=0$, the above boundary conditions turned to be Neumann boundary conditions; it does not affect the species population due to the fully covered environment. Also, rarely, if $\kappa$ takes a larger value $(\longrightarrow \infty)$, then the exterior goes to an entirely unfavorable one. Here, we consider the small value of the unfavorable situation which means some little changes in the environment or in the setting place of species gives an unstable response of species density. To overcome this situation, we consider the control input in the left boundary as

$$
\kappa \vartheta(0, t)-\vartheta_{x}(0, t)=U(t),
$$

where $U(t)$ is an appropriate boundary control.

Furthermore, using a simple transform of variable $\vartheta(x, t)=e^{c_{0} t} w(x, t)$ and appropriate choice of function $f(\vartheta(x, t))$, one can easily see that systems (54)-(57) are equal to systems investigated in this paper. When $U(t)=0$, it gives the unstable response for open loop system (54)-(56). Then, boundary feedback control is to be designed, which helps to overcome the unfavorable situation and achieve the stable response for closed-loop system (54)-(57). So, the proposed results can be implemented to show the stability results for the single-species pattern generation model which is discussed in the following two cases.

Case I: consider the linearized species dynamics

$$
f(\vartheta(x, t))=c_{0} \vartheta(x, t)+c_{1} \vartheta(1, t),
$$

where $c_{0}$ and $c_{1}$ are an arbitrary constants and choose $D=\varepsilon>0$ is the dispersal efficiency. It is noted that the reaction diffusion model (54) with (58) describes a single-species pattern generation with a Dirichlet local term. Now, by considering the transform of variable mentioned above, the reaction diffusion model (54) is equal to parabolic system (1) with Robin boundary conditions and $w(x, t)$ denotes the population density of the species.

Let $\kappa=(1-\alpha / \alpha)$ stands for the value of the unfavorable situation with $0<\alpha<1$, the parameters of the model are $\varepsilon=1, c_{0}=0 \cdot 1, c_{1}=3$, and $L=1$, and the initial population distribution is $w_{0}(x)=1+\sin (3 \pi x / 2)$. Now, using the boundary control input derived in Theorem 1 ,

$$
\begin{aligned}
U(t)= & -(1-\alpha) \frac{c_{1}}{\varepsilon}\left(\frac{1}{s_{1}-s_{2}}\right) \int_{0}^{1}\left[e^{s_{1} y}-e^{s_{2} y}\right] w(y, t) \mathrm{d} y \\
& +\alpha \frac{c_{1}}{\varepsilon}\left(\frac{1}{s_{1}-s_{2}}\right) \int_{0}^{1}\left[-s_{1} e^{s_{1} y}+s_{2} e^{s_{2} y}\right] w(y, t) \mathrm{d} y .
\end{aligned}
$$

For $\alpha=0 \cdot 7$ and $\beta=0 \cdot 7$, we can observe the stable responses of system (1), as shown in Figure 2. Furthermore, the state response of the considered single-species pattern generation model (54)-(58) is presented in Figures 3 and 4. Our main focus here is to study the stability of the species population dynamics (54)-(58) in the presence of a small value of an unfavorable situation. The absence of control actuation gives the divergence results shown in Figure 3. So, from the simulation results, it is easy to observe that the considered model has stable density of the species when the control input is considered; otherwise, there will be an oscillation in the population density of the species. The simulation results for the parabolic system are presented by using finite difference scheme with forward time and central space.

Case II: in this case, we consider the linearized species dynamics as

$$
f(\vartheta(x, t))=c_{0} \vartheta(x, t)+c_{2} \vartheta_{x}(1, t),
$$

where $c_{0}$ and $c_{2}$ are the arbitrary constants. Also, system (54) with (60) describes a single-species pattern generation with the Neumann local term. Following similar lines of Case I, the reaction diffusion model (54) can be described as parabolic system (44) under Robin boundary conditions. 


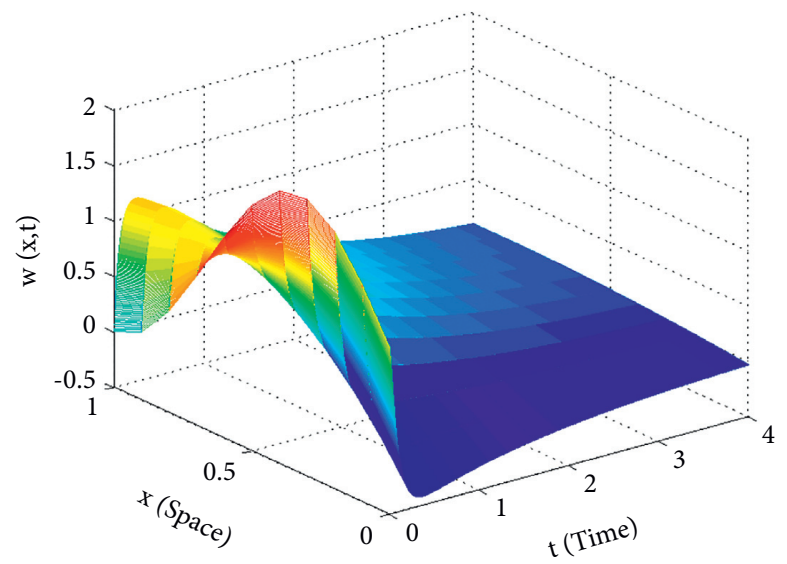

FIGURE 2: $w(x, t)$ of system (1).

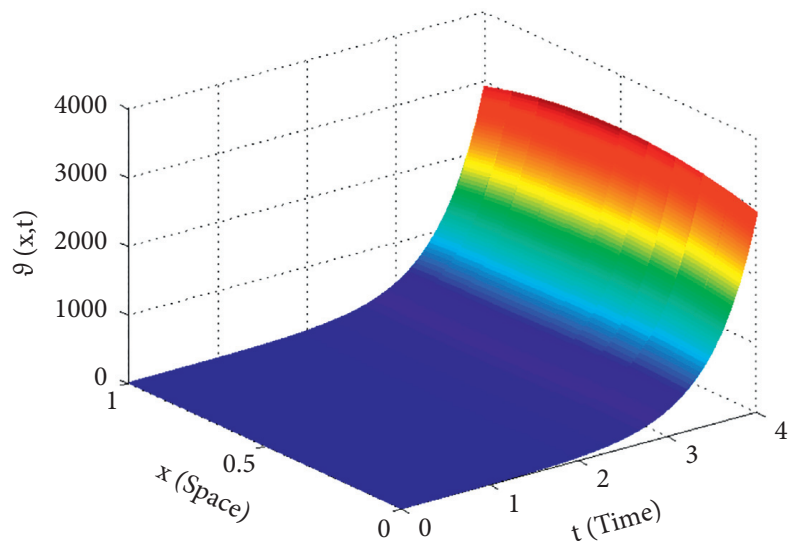

Figure 3: $\vartheta(x, t)$ in Case I without the controller.

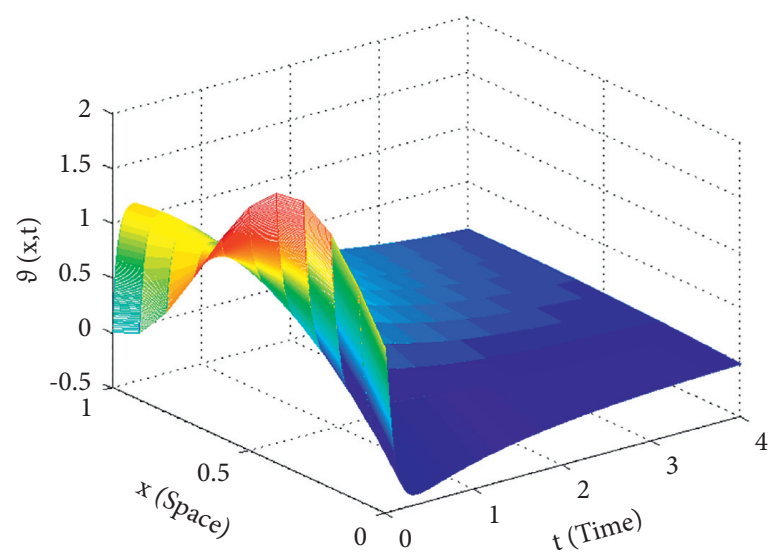

FIgURE 4: $\vartheta(x, t)$ in Case I with the controller. 


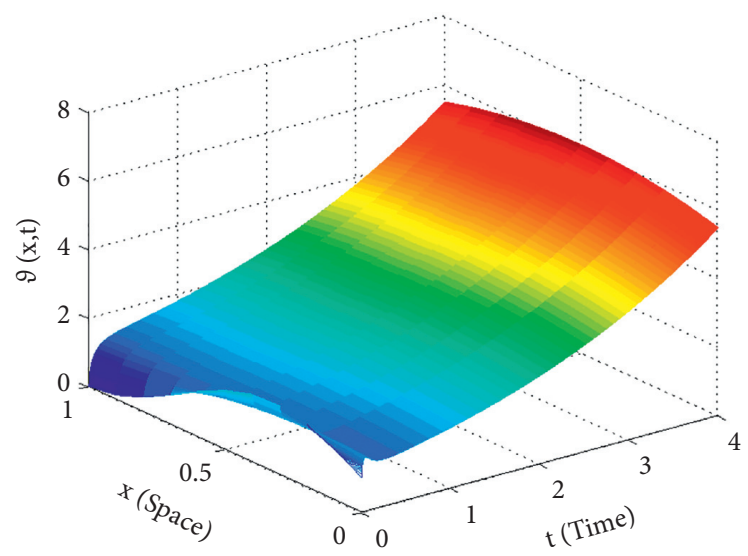

Figure 5: $\vartheta(x, t)$ in Case II without the controller.

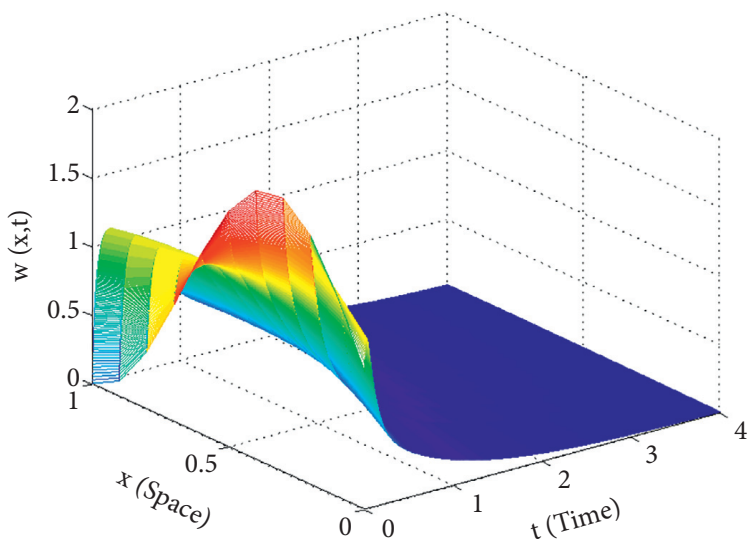

Figure 6: $w(x, t)$ of system (44).

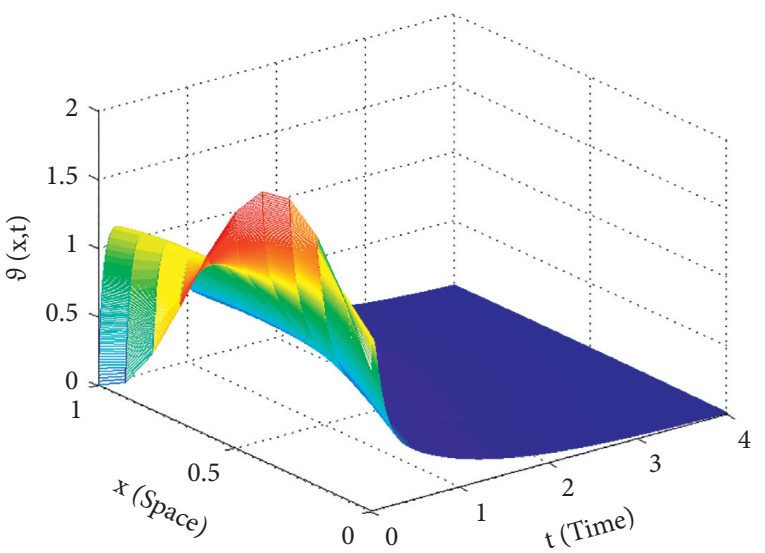

FIGURE 7: $\vartheta(x, t)$ in Case II with the controller. 
Let the parameters $c_{2}=-3, \quad \varepsilon=1, \quad L=1$, and $\kappa=(1-\alpha / \alpha)$, the initial population distribution $w_{0}(x)=1+\sin (3 \pi x / 2)$, and $c_{0}=0 \cdot 1$. Apply the conditions derived in Theorem 4 and the control input,

$$
\bar{U}(t)=(1-\alpha) \frac{c_{2}}{\varepsilon}\left(\frac{1-\beta}{\beta}\right)\left(\frac{1}{s_{3}-s_{4}}\right) \int_{0}^{1}\left[e^{s_{3} y}-e^{s_{4} y}\right] w(y, t) \mathrm{d} y-\alpha \frac{c_{2}}{\varepsilon}\left(\frac{1-\beta}{\beta}\right)\left(\frac{1}{s_{3}-s_{4}}\right) \int_{0}^{1}\left[-s_{3} e^{s_{3} y}+s_{4} e^{s_{4} y}\right] w(y, t) d y .
$$

with $\alpha=0 \cdot 7$ and $\beta=0 \cdot 7$ to model (54). We observe that similar to that of Case I, Figure 5 presents the unstable state response of species population dynamics in the absence of control input. Furthermore, when we consider the control actuation in system (44), systems (54)-(57) with (60) have stable state responses, as shown in Figures 6 and 7 , respectively.

Thus, with the reaction diffusion systems (54)-(57), the population dynamics achieves the stable steady states in the both cases. It is evident that the transform of variable worked well as the simulations of reaction diffusion system (54) is matching in both cases.

Remark 5. It should be noted that using an appropriate transform of variable the problem investigated here can be also useful in some other practical scenarios such as

(i) The general reaction diffusion system with oscillatory kinetics which has the travelling wave-train solution can be modeled [41]

(ii) The PDE model for linearizing a tubular chemical adiabatic reactor [42] derived from the two equations for temperature and concentration when Peclet numbers for heat and mass transfer is equal

\section{Conclusion}

Some new results on the boundary stabilization of the parabolic system with mixed or Robin boundary conditions by using the backstepping method have been investigated. Using Volterra integral transformation, we converted the original system into an exponentially stable target system along with the hyperbolic type of kernel function which has an explicit solution. We have verified the target system is an exponentially stable using Lyapunov stability theory and also demonstrated that the integral transformation is invertible so that the considered system is exponentially stable through the stability of target system. Simulation results of a singlespecies pattern generation model illustrate the effectiveness of the obtained results. The boundary stabilization for class of semilinear fractional PDEs and systems with delays and disturbances is not yet fully studied. In particular, the results involving fractional-order kernel equations are an interesting topic of research; these issues will be focused in our future topics of research.

\section{Data Availability}

The data used to support the findings of this study are included within the article.

\section{Conflicts of Interest}

The authors declare that they have no conflicts of interest.

\section{Acknowledgments}

This work was supported by University Grants Commission (UGC), New Delhi, India, through BSR Start-Up Research Grant.

\section{References}

[1] Q. Wang, H. E. Psillakis, and C. Sun, “Adaptive cooperative control with guaranteed convergence in time-varying networks of nonlinear dynamical systems," IEEE Transactions on Cybernetics, vol. 50, no. 12, pp. 5035-5046, 2020.

[2] Q. Wang and C. Sun, "A continuous distributed control algorithm for time-varying networks of nonlinear agents with input saturation," International Journal of Robust and Nonlinear Control, vol. 31, no. 10, pp. 4616-4628, 2021.

[3] H. Zhang, J. Wang, and J. Gu, "Exponential input-to-state stabilization of an ODE cascaded with a reaction-diffusion equation subject to disturbances," Automatica, vol. 133, Article ID 109885, 2021.

[4] A. Benabdallah, "Stabilization of a class of nonlinear uncertain ordinary differential equation by parabolic partial differential equation controller," International Journal of Robust and Nonlinear Control, vol. 30, no. 7, pp. 3023-3038, 2020.

[5] J. Li, Z. Wu, and C. Wen, "Adaptive stabilization for a reaction-diffusion equation with uncertain nonlinear actuator dynamics," Automatica, vol. 128, Article ID 109594, 2021.

[6] Y. Yuan, Z. Shen, and F. Liao, "Stabilization of coupled ODEPDE system with intermediate point and spatially varying effects interconnection," Asian Journal of Control, vol. 19, no. 3, pp. 1060-1074, 2017.

[7] F. Bribiesca-Argomedo and M. Krstic, "Backstepping-forwarding control and observation for hyperbolic PDEs with Fredholm integrals," IEEE Transactions on Automatic Control, vol. 60, no. 8, pp. 2145-2160, 2015.

[8] J. Wang, M. Krstic, and Y. Pi, "Control of a $2 \times 2$ Coupled linear hyperbolic system sandwiched between 2 ODEs," International Journal of Robust and Nonlinear Control, vol. 28, no. 13, pp. 3987-4016, 2018.

[9] F. Ge, T. Meurer, and Y. Chen, "Mittag-leffler convergent backstepping observers for coupled semilinear subdiffusion systems with spatially varying parameters," Systems and Control Letters, vol. 122, pp. 86-92, 2018.

[10] Y. Si and C. Xie, "Local stabilization of semilinear parabolic system by boundary control," Asian Journal of Control, vol. 23, no. 1, pp. 591-599, 2019.

[11] C. Wei and J. Li, “A generalized exponential stabilization for a class of semilinear parabolic equations: linear boundary feedback control approach," Mathematical Methods in the Applied Sciences, vol. 44, no. 18, Article ID 14677, 2021. 
[12] D. Colton, "Integral operators and reflection principles for parabolic equations in one space variable," Journal of Differential Equations, vol. 15, no. 3, pp. 551-559, 1974.

[13] D. Colton, "The solution of initial-boundary value problems for parabolic equations by the method of integral operators," Journal of Differential Equations, vol. 26, no. 2, pp. 181-190, 1977.

[14] R. Triggiani, "Boundary feedback stabilizability of parabolic equations," Applied Mathematics and Optimization, vol. 6, no. 1, pp. 201-220, 1980.

[15] T. I. Seidman, "Two results on exact boundary control of parabolic equations," Applied Mathematics and Optimization, vol. 11, no. 1, pp. 145-152, 1984.

[16] P. V. Kokotovic, "the joy of feedback: nonlinear and adaptive," IEEE Control Systems Magazine, vol. 12, no. 3, pp. 7-17, 1992.

[17] R. Lozano and B. Brogliato, "Adaptive control of robot manipulators with flexible joints," IEEE Transactions on Automatic Control, vol. 37, no. 2, pp. 174-181, 1992.

[18] M. Krstic and A. Smyshlyaev, "Boundary control of PDEs," A Course on Backstepping Designs, SIAM, Bangkok, Thailand, 2008.

[19] R. Altmann, "A PDAE formulation of parabolic problems with dynamic boundary conditions," Applied Mathematics Letters, vol. 90, pp. 202-208, 2019.

[20] W. Liu, "Boundary feedback stabilization of an unstable heat equation," SIAM Journal on Control and Optimization, vol. 42, no. 3, pp. 1033-1043, 2003.

[21] J. Wang, "The stability of equilibria for a reaction-diffusionODE system on convex domains," Applied Mathematics Letters, vol. 93, pp. 147-152, 2019.

[22] A. Smyshlyaev and M. Krstic, "On control design for PDEs with space-dependent diffusivity or time-dependent reactivity," Automatica, vol. 41, no. 9, pp. 1601-1608, 2005.

[23] T. Hashimoto and M. Krstic, "Stabilization of reaction diffusion equations with state delay using boundary control input," IEEE Transactions on Automatic Control, vol. 61, no. 12, pp. 4041-4047, 2016.

[24] J. Auriol, K. A. Morris, and F. Di Meglio, "Late-lumping backstepping control of partial differential equations," Automatica, vol. 100, pp. 247-259, 2019.

[25] D. M. Boskovic and M. Krstic, "Stabilization of a solid propellant rocket instability by state feedback," International Journal of Robust and Nonlinear Control, vol. 13, no. 5, pp. 483-495, 2003.

[26] R. Vazquez and M. Krstic, "Boundary control and estimation of reaction-diffusion equations on the sphere under revolution symmetry conditions," International Journal of Control, vol. 92, no. 1, pp. 2-11, 2017.

[27] N. Ghaderi and M. Keyanpour, "Backstepping design for a class of coupled parabolic PDEs with spatially varying coefficient," Asian Journal of Control, vol. 22, no. 5, pp. 2017-2028, 2019.

[28] S. Chen, R. Vazquez, and M. Krstic, "Folding backstepping approach to parabolic PDE bilateral boundary control," IFACPapersOnLine, vol. 52, no. 2, pp. 76-81, 2019.

[29] A. Alessandri, P. Bagnerini, R. Cianci, S. Donnarumma, and A. Taddeo, "Stabilization of diffusive systems using backstepping and the circle criterion," International Journal of Heat and Mass Transfer, vol. 149, Article ID 119132, 2020.

[30] N. Espitia, A. Polyakov, D. Efimov, and W. Perruquetti, "Boundary time-varying feedbacks for fixed-time stabilization of constant-parameter reaction-diffusion systems," Automatica, vol. 103, pp. 398-407, 2019.
[31] J. Qi, M. Krstic, and S. Wang, "Stabilization of reactiondiffusions PDE with delayed distributed actuation," Systems and Control Letters, vol. 133, Article ID 104558, 2019.

[32] M. Li and W. Mao, "Finite-time bounded control for coupled parabolic PDE-ODE systems subject to boundary disturbances," Mathematical Problems in Engineering, vol. 2020, Article ID 8882382, 13 pages, 2020.

[33] S. Fan, "A new general decay rate of wave equation with memory-type boundary control," Mathematical Problems in Engineering, vol. 2021, Article ID 5571072, 11 pages, 2021.

[34] M. Ghattassi and T. M. Laleg, "Boundary stabilization of a reaction-diffusion system weakly coupled at the boundary," IFAC-PapersOnLine, vol. 53, no. 2, Article ID 16537, 2020.

[35] C. Bao, B. Cui, X. Lou, W. Wu, and B. Zhuang, "fixed-time stabilisation of boundary controlled linear parabolic distributed parameter systems with space-dependent reactivity," IET Control Theory and Applications, vol. 15, no. 11, pp. 652-667, 2020.

[36] T. R. Oliveira, J. Feiling, S. Koga, and M. Krstic, "extremum seeking for unknown scalar maps in cascade with a class of parabolic partial differential equations," International Journal of Adaptive Control and Signal Processing, vol. 35, no. 7, pp. 1-26, 2020.

[37] H. Anfinsen and O. M. Aamo, "Stabilization and tracking control of a time-variant linear hyperbolic PIDE using backstepping," Automatica, vol. 116, Article ID 108929, 2020.

[38] W. L. J. Wang and W. Guo, "A backstepping approach to adaptive error feedback rgulator design for one-dimensional linear parabolic PIDEs," Journal of Mathematical Analysis and Applications, vol. 503, no. 2, Article ID 125310, 2021.

[39] A. Smyshlyaev and M. Krstic, "closed form boundary state feedbacks for a class of 1D partial integro-differential equations," IEEE Transactions on Automatic Control, vol. 49, pp. 1557-1564, 2004.

[40] G. H. Hardy, J. E. Littlewood, and G. Polya, Inequalities, Cambridge University Press, Cambridge, UK, 2nd edition, 1959.

[41] J. D. Murray, "Mathematical biology," II: Spatial Models and Biomedical Applications, Springer, Heidelberg, Germany, 3rd edition, 2003

[42] D. M. Boskovic, A. Balogh, and M. Krstic, "Backstepping in infinite dimension for a class of parabolic distributed parameter systems," Mathematics of Control, Signals and Systems, vol. 16, pp. 44-75, 2003. 